QUARTERLY OF APPLIED MATHEMATICS

VOLUME LXVIII, NUMBER 1

MARCH 2010, PAGES 73-80

S 0033-569X(09)01142-1

Article electronically published on October 20, 2009

\title{
A FLUID DYNAMIC FORMULATION OF THE ISOMETRIC EMBEDDING PROBLEM IN DIFFERENTIAL GEOMETRY
}

\author{
BY \\ GUI-QIANG CHEN (Department of Mathematics, Northwestern University, Evanston, Illinois \\ 60208), \\ MARSHALL SLEMROD (Department of Mathematics, University of Wisconsin, Madison, \\ Wisconsin 53706), \\ AND \\ DEHUA WANG (Department of Mathematics, University of Pittsburgh, Pittsburgh, Pennsylvania \\ 15260) \\ Dedicated to Walter Strauss on the occasion of his 70th birthday
}

\begin{abstract}
The isometric embedding problem is a fundamental problem in differential geometry. A longstanding problem is considered in this paper to characterize intrinsic metrics on a two-dimensional Riemannian manifold which can be realized as isometric immersions into the three-dimensional Euclidean space. A remarkable connection between gas dynamics and differential geometry is discussed. It is shown how the fluid dynamics can be used to formulate a geometry problem. The equations of gas dynamics are first reviewed. Then the formulation using the fluid dynamic variables in conservation laws of gas dynamics is presented for the isometric embedding problem in differential geometry.
\end{abstract}

1. Introduction. We are concerned with isometric embeddings or immersions (i.e., realizations) of two-dimensional Riemannian manifolds in the Euclidean space $\mathbb{R}^{3}$. A classical question in differential geometry is whether one can isometrically embed a Riemannian manifold $\left(\mathcal{M}^{n}, g\right)$ into $\mathbb{R}^{N}$ for $N$ large enough. Nash 15 indicated that any smooth compact manifold $\left(\mathcal{M}^{n}, g\right)$ can always be isometrically embedded into $\mathbb{R}^{3 s_{n}+4 n}$ for $s_{n}=n(n-1) / 2$. This important paper laid the foundation for the development of geometric analysis in the second half of the 20th century. Gromov [8] proved that one

Received August 6, 2008.

2000 Mathematics Subject Classification. Primary 35M10, 76H05, 76N10, 76L05, 53C42.

Key words and phrases. Isometric embedding, two-dimensional Riemannian manifold, differential geometry, transonic flow, gas dynamics, viscosity method, compensated compactness.

E-mail address: gqchen@math.northwestern.edu

E-mail address: slemrod@math.wisc.edu

E-mail address: dwang@math.pitt.edu 
can embed any $\left(\mathcal{M}^{n}, g\right)$ even into $\mathbb{R}^{s_{n}+2 n+3}$. Then a further natural question is to find the smallest dimension $N(n)$ for the Riemannian manifold $\left(\mathcal{M}^{n}, g\right)$ to be isometrically embeddable in $\mathbb{R}^{N(n)}$. In particular, a fundamental, longstanding open problem is to characterize intrinsic metrics on a two-dimensional Riemannian manifold $\mathcal{M}^{2}$ which can be realized as isometric immersions into $\mathbb{R}^{3}$ (cf. [9, 16, 17, 20] and the references cited therein). Important results have been achieved for the embedding of surfaces with positive Gauss curvature which can be formulated as an elliptic boundary value problem (cf. [9]). For the case of surfaces of negative Gauss curvature where the underlying partial differential equations are hyperbolic, the complementary problem would be an initial or initial-boundary value problem. When the Gauss curvature changes sign, the problem then becomes an initial-boundary value problem of mixed elliptic-hyperbolic type. Hong in [10] first proved that complete negatively curved surfaces can be isometrically immersed in $\mathbb{R}^{3}$ if the Gauss curvature decays at a certain rate in the time-like direction. In fact, a crucial lemma in Hong [10] (also see Lemma 10.2.9 in 9]) shows that, for such a decay rate of the negative Gauss curvature, there exists a unique global smooth, small solution forward in time for prescribed smooth, small initial data. We are interested in solving the corresponding problem for a class of large non-smooth initial data.

In Chen-Slemrod-Wang [3], we have introduced a general approach, which combines a fluid dynamic formulation of balance laws with a compensated compactness framework, to deal with the isometric immersion problem in $\mathbb{R}^{3}$ (even when the Gauss curvature changes sign). In Chen-Slemrod-Wang [2, we have developed a vanishing viscosity method to establish the existence of a weak entropy solution to the transonic flow in gas dynamics past an obstacle such as an airfoil, via the method of compensated compactness $(14,[18])$. We have found in [3] that the idea of [2] for gas dynamics is useful for solving the isometric embedding problem in differential geometry. In particular, in $[\underline{3}$, we have formulated the isometric immersion problem for two-dimensional Riemannian manifolds in $\mathbb{R}^{3}$ via solvability of the Gauss-Codazzi system, and we have introduced a fluid dynamic formulation of balance laws for the Gauss-Codazzi system. Then we have formed a compensated compactness framework and will present one of our main observations that this framework is a natural formulation to ensure the weak continuity of the Gauss-Codazzi system for approximate solutions, which yields the isometric realization of two-dimensional surfaces in $\mathbb{R}^{3}$. As a first application of this approach, we have focused on the isometric immersion problem of two-dimensional Riemannian manifolds with strictly negative Gauss curvature. Since the local existence of smooth solutions follows from the standard hyperbolic theory, we are concerned with the global existence of solutions of the initial value problem with large initial data. The metrics $\left(g_{i j}\right)$ we study have special structures and forms that are usually associated with the catenoid of revolution. For these cases, while Hong's theorem [10] applies to obtain the existence of a solution for small smooth initial data, our result yields a large-data existence theorem for a $C^{1,1}$ isometric immersion. To achieve this, we have introduced a vanishing viscosity method depending on the features of the initial value problem for isometric immersions and have presented a technique to make the apriori estimates, including the $L^{\infty}$ control and $H^{-1}$-compactness for the viscous approximate solutions. This yields the weak convergence of the vanishing viscosity approximate solutions and the weak continuity 
of the Gauss-Codazzi system for the approximate solutions, hence the existence of a $C^{1,1}$-isometric immersion of the manifold into $\mathbb{R}^{3}$ with prescribed initial conditions.

From Chen-Slemrod-Wang 2, 3, we have seen a remarkable connection between the two distinct areas of gas dynamics and differential geometry. Here we present such a connection and show how the fluid dynamics can be used to formulate a geometry problem. Thus, we will present first the equations in Chen-Slemrod-Wang [2] for the transonic flow problem in gas dynamics, and then the formulation using the fluid dynamic variables in conservation laws of gas dynamics for the isometric embedding problem in differential geometry.

2. Equations of gas dynamics. In two space dimensions with variables $(x, y)$, the steady transonic flow of the isentropic case is governed by the following steady Euler equations on conservations of mass and momentum in gas dynamics:

$$
\left\{\begin{array}{l}
(\rho u)_{x}+(\rho v)_{y}=0 \\
\left(\rho u^{2}+p\right)_{x}+(\rho u v)_{y}=0 \\
(\rho u v)_{x}+\left(\rho v^{2}+p\right)_{y}=0
\end{array}\right.
$$

where $\rho$ is the density, $(u, v)$ is the velocity, and $p=\frac{\rho^{\gamma}}{\gamma}(\gamma \geq 1)$ is the pressure. If we assume that the flow is irrotational, then system (2.1) can be reduced to the following two equations of irrotationality and conservation of mass:

$$
\left\{\begin{array}{l}
v_{x}-u_{y}=0 \\
(\rho u)_{x}+(\rho v)_{y}=0
\end{array}\right.
$$

and, by scaling, the density $\rho$ is determined by Bernoulli's law:

$$
\rho=\left(1-\frac{\gamma-1}{2} q^{2}\right)^{\frac{1}{\gamma-1}}
$$

where $q$ is the flow speed defined by $q^{2}=u^{2}+v^{2}$. The sound speed $c$ is defined as

$$
c^{2}=p^{\prime}(\rho)=1-\frac{\gamma-1}{2} q^{2} .
$$

At the cavitation point $\rho=0$,

$$
q=q_{c a v}:=\sqrt{\frac{2}{\gamma-1}} .
$$

At the stagnation point $q=0$, the density reaches its maximum $\rho=1$. Bernoulli's law (2.3) is valid for $0 \leq q \leq q_{c a v}$. At the sonic point $q=c$, (2.4) implies $q^{2}=\frac{2}{\gamma+1}$. Define the critical speed $q_{c r}$ as

$$
q_{c r}:=\sqrt{\frac{2}{\gamma+1}} .
$$

We rewrite Bernoulli's law (2.3) in the form

$$
q^{2}-q_{c r}^{2}=\frac{2}{\gamma+1}\left(q^{2}-c^{2}\right) .
$$


Thus the flow is subsonic when $q<q_{c r}$, sonic when $q=q_{c r}$, and supersonic when $q>q_{c r}$. For the isothermal flow $(\gamma=1), p=c^{2} \rho$, where $c>0$ is the constant sound speed, the density $\rho$ is given by Bernoulli's law:

$$
\rho=\rho_{0} \exp \left(-\frac{u^{2}+v^{2}}{2 c^{2}}\right)
$$

for some constant $\rho_{0}>0$, and $q_{c r}=c$.

3. Isometric embedding in differential geometry. In this section, we discuss the isometric embedding problem in differential geometry in $\mathbb{R}^{3}$ and its formulation of fluid dynamics.

We first give the Gauss-Codazzi system of isometric embedding in $\mathbb{R}^{3}$. Let $g_{i j}, i, j=$ 1,2 , be the given metric of a two-dimensional Riemannian manifold $\mathcal{M}$ parameterized on an open set $\Omega \subset \mathbb{R}^{2}$. The first fundamental form $I$ for $\mathcal{M}$ on $\Omega$ is

$$
I:=g_{11}(d x)^{2}+2 g_{12} d x d y+g_{22}(d y)^{2},
$$

and the isometric embedding problem is to seek a map $\mathbf{r}: \Omega \rightarrow \mathbb{R}^{3}$ such that $d \mathbf{r} \cdot d \mathbf{r}=I$. That is,

$$
\partial_{x} \mathbf{r} \cdot \partial_{x} \mathbf{r}=g_{11}, \quad \partial_{x} \mathbf{r} \cdot \partial_{y} \mathbf{r}=g_{12}, \quad \partial_{y} \mathbf{r} \cdot \partial_{y} \mathbf{r}=g_{22},
$$

so that $\left\{\partial_{x} \mathbf{r}, \partial_{y} \mathbf{r}\right\}$ in $\mathbb{R}^{3}$ are linearly independent. The corresponding second fundamental form is

$$
I I:=h_{11}(d x)^{2}+2 h_{12} d x d y+h_{22}(d y)^{2} .
$$

The fundamental theorem of surface theory (cf. 4, 9]) indicates that there exists a surface in $\mathbb{R}^{3}$ whose first and second fundamental forms are $I$ and $I I$ if the coefficients $\left(g_{i j}\right)$ and $\left(h_{i j}\right)$ of the two given quadratic forms $I$ and $I I$ with $\left(g_{i j}\right)>0$ satisfy the Gauss-Codazzi system. It is indicated in Mardare [13] (Theorem 9; also see [12]) that this theorem holds even when $\left(h_{i j}\right)$ is only in $L^{\infty}$ for given $\left(g_{i j}\right)$ in $C^{1,1}$, for which the immersion surface is $C^{1,1}$. This shows that, for the realization of a two-dimensional Riemannian manifold in $\mathbb{R}^{3}$ with given metric $\left(g_{i j}\right)>0$, it suffices to solve $\left(h_{i j}\right) \in L^{\infty}$ determined by the Gauss-Codazzi system to recover $\mathbf{r}$ a posteriori. The Gauss-Codazzi system (cf. [4, 9]) can be written as

$$
\left\{\begin{array}{l}
\partial_{x} M-\partial_{y} L=\Gamma_{22}^{(2)} L-2 \Gamma_{12}^{(2)} M+\Gamma_{11}^{(2)} N \\
\partial_{x} N-\partial_{y} M=-\Gamma_{22}^{(1)} L+2 \Gamma_{12}^{(1)} M-\Gamma_{11}^{(1)} N
\end{array}\right.
$$

with

$$
L N-M^{2}=\kappa,
$$

where

$$
L=\frac{h_{11}}{\sqrt{|g|}}, \quad M=\frac{h_{12}}{\sqrt{|g|}}, \quad N=\frac{h_{22}}{\sqrt{|g|}}, \quad|g|=\operatorname{det}\left(g_{i j}\right)=g_{11} g_{22}-g_{12}^{2},
$$

$\kappa(x, y)$ is the Gauss curvature that is determined by the relation

$$
\kappa(x, y)=\frac{R_{1212}}{|g|}, R_{i j k l}=g_{l m}\left(\partial_{k} \Gamma_{i j}^{(m)}-\partial_{j} \Gamma_{i k}^{(m)}+\Gamma_{i j}^{(n)} \Gamma_{n k}^{(m)}-\Gamma_{i k}^{(n)} \Gamma_{n j}^{(m)}\right),
$$


$R_{i j k l}$ is the curvature tensor and depends on $\left(g_{i j}\right)$ and its first and second derivatives, and

$$
\Gamma_{i j}^{(k)}=\frac{1}{2} g^{k l}\left(\partial_{j} g_{i l}+\partial_{i} g_{j l}-\partial_{l} g_{i j}\right)
$$

is the Christoffel symbol and depends on the first derivatives of $\left(g_{i j}\right)$, where the summation convention is used, $\left(g^{k l}\right)$ denotes the inverse of $\left(g_{i j}\right)$, and $\left(\partial_{1}, \partial_{2}\right)=\left(\partial_{x}, \partial_{y}\right)$. Therefore, given a positive definite metric $\left(g_{i j}\right) \in C^{1,1}$, the Gauss-Codazzi system gives us three equations for the three unknowns $(L, M, N)$ determining the second fundamental form $I I$. Note that, although $\left(g_{i j}\right)$ is positive definite, $R_{1212}$ may change sign, and so does the Gauss curvature $\kappa$. Thus, the Gauss-Codazzi system (3.1)-(3.2) generically is of mixed hyperbolic-elliptic type, as in transonic flow (cf. [2]). In Chen-Slemrod-Wang [3], we have introduced a general approach to deal with the isometric immersion problem involving nonlinear partial differential equations of mixed hyperbolic-elliptic type by combining a fluid dynamic formulation of balance laws with a compensated compactness framework. As an example of direct applications of this approach, we have shown how this approach can be applied to establish an isometric immersion of a two-dimensional Riemannian manifold with negative Gauss curvature in $\mathbb{R}^{3}$.

We now describe the fluid dynamic formulation of the Gauss-Codazzi system (3.1)(3.2) in detail. Although, from the viewpoint of geometry, the constraint condition (3.2) is a Monge-Ampère equation and the equations in (3.1) are integrability relations, we can put the problem into a fluid dynamic formulation so that the isometric immersion problem may be solved via the approaches for transonic flows of fluid dynamics in ChenSlemrod-Wang [2]. To do this, we set

$$
L=\rho v^{2}+p, \quad M=-\rho u v, \quad N=\rho u^{2}+p,
$$

and set $q^{2}=u^{2}+v^{2}$ as usual. Then the equations in (3.1) become the familiar balance laws of momentum:

$$
\left\{\begin{array}{l}
\partial_{x}(\rho u v)+\partial_{y}\left(\rho v^{2}+p\right)=-\left(\rho v^{2}+p\right) \Gamma_{22}^{(2)}-2 \rho u v \Gamma_{12}^{(2)}-\left(\rho u^{2}+p\right) \Gamma_{11}^{(2)}, \\
\partial_{x}\left(\rho u^{2}+p\right)+\partial_{y}(\rho u v)=-\left(\rho v^{2}+p\right) \Gamma_{22}^{(1)}-2 \rho u v \Gamma_{12}^{(1)}-\left(\rho u^{2}+p\right) \Gamma_{11}^{(1)},
\end{array}\right.
$$

and the Monge-Ampère constraint (3.2) becomes

$$
\rho p q^{2}+p^{2}=\kappa .
$$

We choose pressure $p$ as for the Chaplygin-type gas:

$$
p=-\frac{1}{\rho} .
$$

Then, from (3.4), we have the "Bernoulli" relation:

$$
\rho=\frac{1}{\sqrt{q^{2}+\kappa}} .
$$

This yields

$$
p=-\sqrt{q^{2}+\kappa},
$$

and the formulas for $u^{2}$ and $v^{2}$ :

$$
u^{2}=p(p-M), \quad v^{2}=p(p-L), \quad M^{2}=(N-p)(L-p) .
$$


The last relation for $M^{2}$ gives the relation for $p$ in terms of $(L, M, N)$, and then the first two give the relations for $(u, v)$ in terms of $(L, M, N)$.

We rewrite (3.3) as

$$
\left\{\begin{array}{l}
\partial_{x}(\rho u v)+\partial_{y}\left(\rho v^{2}+p\right)=R_{1}, \\
\partial_{x}\left(\rho u^{2}+p\right)+\partial_{y}(\rho u v)=R_{2},
\end{array}\right.
$$

where $R_{1}$ and $R_{2}$ denote the right-hand sides of (3.3). Then we can write our "rotationality-continuity equations" as

$$
\left\{\begin{array}{l}
\partial_{x} v-\partial_{y} u=\frac{1}{\rho q^{2}}\left(u\left(\frac{1}{2} \rho \partial_{y} \kappa+R_{1}\right)-v\left(\frac{1}{2} \rho \partial_{x} \kappa+R_{2}\right)\right), \\
\partial_{x}(\rho u)+\partial_{y}(\rho v)=\frac{1}{2} \frac{\rho u}{q^{2}} \partial_{x} \kappa+\frac{1}{2} \frac{\rho v}{q^{2}} \partial_{y} \kappa+\frac{v}{q^{2}} R_{1}+\frac{u}{q^{2}} R_{2} .
\end{array}\right.
$$

In summary, the Gauss-Codazzi system (3.1)-(3.2), the momentum equations (3.3)-(3.6), and the rotationality-continuity equations (3.5) and (3.8) are all formally equivalent. However, for weak solutions, we know from our experience with gas dynamics that this equivalence breaks down. In Chen-Dafermos-Slemrod-Wang [1, the decision has been made (as is standard in gas dynamics) to solve the rotationality-continuity equations and view the momentum equations as "entropy" equalities which may become inequalities for weak solutions. In geometry, this situation is just the reverse. It is the Gauss-Codazzi system that must be solved exactly, and hence the rotationality-continuity equations will become "entropy" inequalities for weak solutions.

We define the "sound" speed as

$$
c^{2}=p^{\prime}(\rho)=\frac{1}{\rho^{2}}
$$

then from our "Bernoulli" relation (3.5) we see

$$
c^{2}=q^{2}+\kappa .
$$

Hence, under this formulation,

(i) when $\kappa>0$, the "flow" is subsonic, i.e., $q<c$, and system (3.3)-3.4 is elliptic;

(ii) when $\kappa<0$, the "flow" is supersonic, i.e., $q>c$, and system (3.3)-(3.4) is hyperbolic;

(iii) when $\kappa=0$, the "flow" is sonic, i.e., $q=c$, and system (3.3)-3.4) is degenerate.

In general, system (3.3)-(3.4) is of mixed hyperbolic-elliptic type. Thus, the isometric immersion problem involves the existence of solutions to nonlinear partial differential equations of mixed hyperbolic-elliptic type.

In Chen-Slemrod-Wang [3], we have considered one of the spatial variables $x$ and $y$ as time-like, have introduced a vanishing viscosity method via parabolic regularization to obtain the uniform $L^{\infty}$ estimate by identifying invariant regions for the approximate solutions, and have shown that the $H_{l o c}^{-1}$-compactness can be achieved for the viscous approximate solutions. Then, as in Chen-Slemrod-Wang [2], the compensated compactness framework yields a weak solution to the initial value problem of system (3.3)-(3.4) when the initial data lies in the diamond-shaped invariant region. This establishes a $C^{1,1}\left(\mathbb{R}^{2}\right)$ immersion of the Riemannian manifold into $\mathbb{R}^{3}$. In particular, our existence result asserts the existence of a $C^{1,1}$-surface for the associated metric for a class of non-circular 
cross-sections prescribed at $x=0$ for catenoid. See [3] for the details. Possible implication of our approach may be in existence theorems for equilibrium configurations of a catenoidal shell as detailed in Vaziri-Mahedevan [19]. However, the existence of isometric embeddings/immersions of a general surface with negative Gauss curvature is still open. When the Gauss curvature $\kappa$ changes sign, the problem becomes transonic and thus a mixed hyperbolic-elliptic type. In this mixed-type problem, only special local solutions are known to exist for special data $([11,9])$, and the existence of global solutions is a significantly difficult open problem.

Acknowledgments. The first author's research was supported in part by the National Science Foundation under Grants DMS-0807551, DMS-0720925, and DMS0505473. The second author's research was supported in part by the National Science Foundation under Grant DMS-0647554. The third author's research was supported in part by the National Science Foundation under Grant DMS-0604362, and by the Office of Naval Research under Grant N00014-07-1-0668.

\section{REFERENCES}

[1] G.-Q. Chen, C. Dafermos, M. Slemrod, and D. Wang, On two-dimensional sonic-subsonic flow, Commun. Math. Phys. 271 (2007), 635-647. MR2291790 (2008e:35149)

[2] G.-Q. Chen, M. Slemrod, and D. Wang, Vanishing viscosity method for transonic flow, Arch. Rational Mech. Anal. 189 (2008), 159-188. MR2403603

[3] G.-Q. Chen, M. Slemrod, and D. Wang, Isometric immersions and compensated compactness, submitted.

[4] M. P. do Carmo, Riemannian Geometry, Transl. by F. Flaherty, Birkhäuser: Boston, MA, 1992. MR.1138207 (92i:53001)

[5] G.-C. Dong, Nonlinear Partial Differential Equations of Second Order, Translations of Mathematical Monographs, 95, American Mathematical Society, Providence, RI, 1991.

[6] R. Finn and D. Gilbarg, Uniqueness and the force formulas for plane subsonic flows, Trans. Amer. Math. Soc. 88 (1958), 375-379.

[7] F. I. Frankl, M. V. Keldysh, Die äussere Neumann'sche Aufgabe für nichtlineare elliptische Differentialgleichungen mit Anwendung auf die Theorie der Flügel im kompressiblem Gas (Russian, German summary), Izvestiya Akademii Nauk SSR, Series 7 (1934), no. 4, 561-607.

[8] M. Gromov, Partial Differential Relations, Springer-Verlag: Berlin, 1986. MR864505 (90a:58201)

[9] Q. Han, and J.-X. Hong, Isometric Embedding of Riemannian Manifolds in Euclidean Spaces, AMS: Providence, RI, 2006. MR2261749 (2008e:53055)

[10] J.-X. Hong, Realization in $\mathbb{R}^{3}$ of complete Riemannian manifolds with negative curvature, Comm. Anal. Geom. 1 (1993), no. 3-4, 487-514. MR1266477 (95d:53003)

[11] C.-S. Lin, The local isometric embedding in $\mathbb{R}^{3}$ of 2-dimensional Riemannian manifolds with Gaussian curvature changing sign cleanly, Comm. Pure Appl. Math. 39 (1986), 867-887. MR0859276 (88e:53097)

[12] S. Mardare, The fundamental theorem of surface theory for surfaces with little regularity, J. Elasticity 73 (2003), 251-290. MR2057747 (2005c:53003)

[13] S. Mardare, On Pfaff systems with $L^{p}$ coefficients and their applications in differential geometry, J. Math. Pure Appl. 84 (2005), 1659-1692. MR2180386 (2006k:58003)

[14] F. Murat, Compacite par compensation, Ann. Suola Norm. Pisa (4), 5 (1978), 489-507. MR506997 (80h:46043a)

[15] J. Nash, The imbedding problem for Riemannian manifolds, Ann. Math. (2), 63, 20-63. MR0075639 $(17: 782 \mathrm{~b})$

[16] Ė. G. Poznyak and E. V. Shikin, Small parameters in the theory of isometric imbeddings of twodimensional Riemannian manifolds in Euclidean spaces. In: Some Questions of Differential Geometry in the Large, Amer. Math. Soc. Transl. Ser. 2, 176 (1996), 151-192, AMS: Providence, RI. MR:1406844(98c:53006) 
[17] È. R. Rozendorn, Surfaces of negative curvature. In: Geometry, III, 87-178, 251-256, Encyclopaedia Math. Sci. 48, Springer: Berlin, 1992. MR1306735

[18] L. Tartar, Compensated compactness and applications to partial differential equations. In: Nonlinear Analysis and Mechanics, Heriot-Watt Symposium IV, Res. Notes in Math. 39, pp. 136-212, Pitman: Boston-London, 1979. MR 584398 (81m:35014)

[19] A. Vaziri, and L. Mahedevan, Localized and extended deformations of elastic shells, Proc. National Acad. Sci, USA 105 (2008), 7913-7918.

[20] S.-T. Yau, Review of geometry and analysis. In: Mathematics: Frontiers and Perspectives, pp. 353401, International Mathematics Union, Eds. V. Arnold, M. Atiyah, P. Lax, and B. Mazur, AMS: Providence, 2000. MR 1754787(2001m:53003) 